\title{
Tissue-specific expression of p73 and p63 isoforms in human tissues
}

\author{
Clayton B. Marshall (iD ${ }^{1,6}$, J. Scott Beeler (iD ${ }^{2,6}$, Brian D. Lehmann (DiD ${ }^{2,3}$, Paula Gonzalez-Ericsson ${ }^{3}$, Violeta Sanchez ${ }^{3}$, \\ Melinda E. Sanders ${ }^{2,3,4}$, Kelli L. Boyd ${ }^{5}$ and Jennifer A. Pietenpol ${ }^{1,2,3}$
}

(c) The Author(s) 2021

p73 and p63 are members of the p53 family that exhibit overlapping and distinct functions in development and homeostasis. The evaluation of p73 and p63 isoform expression across human tissue can provide greater insight to the functional interactions between family members. We determined the mRNA isoform expression patterns of TP73 and TP63 across a panel of 36 human tissues and protein expression within the highest-expressing tissues. TP73 and TP63 expression significantly correlated across tissues. In tissues with concurrent mRNA expression, nuclear co-expression of both proteins was observed in a majority of cells. Using GTEx data, we quantified p73 and p63 isoform expression in human tissue and identified that the a-isoforms of TP73 and TP63 were the predominant isoform expressed in nearly all tissues. Further, we identified a previously unreported p73 mRNA product encoded by exons 4 to 14. In sum, these data provide the most comprehensive tissue-specific atlas of p73 and p63 protein and mRNA expression patterns in human and murine samples, indicating coordinate expression of these transcription factors in the majority of tissues in which they are expressed.

Cell Death and Disease (2021)12:745; https://doi.org/10.1038/s41419-021-04017-8

\section{INTRODUCTION}

p73 and p63 are members of the p53 family of sequence-specific transcription factors that directly regulate differentiation, cell cycle, proliferation, DNA repair, and apoptosis [1-5]. p73 and p63 bind similar consensus DNA binding sites $[6,7]$; but diverge in their regulation of gene targets [8-11]. Further, p73 and p63 can heterodimerize to differentially co-regulate downstream target genes [12-15].

The functional and physical interactions between p73 and p63 lead to complicated, multi-faceted regulation of target genes. p73 is co-expressed with p63 in the basal cell populations of many epithelial tissues [16]. Previously, we discovered that $50 \%$ of basal epithelial cells in the trachea express p73 and that the tracheas from p73-deficient (p73-/-) mice have a 35\% reduction of basal cells in addition to a loss of multiciliated epithelial cells [17]. p73 protein expression is a marker of the basal epidermal stem cell populations located near hair follicles in scale-like skin [18]. Further, our laboratory found that p73-/- mice have delayed epidermal wound healing attributable to regulation of genes involved in proliferation, DNA damage response, cellular junctions, and skin development [19]. These results collectively suggest a role for p73 in basal epithelial cells, and highlight the need to examine further the coordinate isoform expression of p73 and p63 across human tissues.

Both p73 and p63 genes have two promoters (P1 and P2) that give rise to isoforms with distinct transcriptional programs $[1,5,9,20-23]$. Isoforms transcribed from P1 contain a full- length transactivation (TA) domain [1, 5], while isoforms transcribed from P2 have a truncated TA $(\Delta N)$ domain $[5,20]$. The TP73 and TP63 genes also undergo C-terminal alternative splicing, which generates several isoforms (termed $\alpha, \beta, \gamma$, etc.) with different activity at target genes [1, 5, 24, 25]. p73 has also been reported to undergo $\mathrm{N}$-terminal alternative splicing that removes exons from the TA domain $(\Delta \mathrm{ex} 2$ and $\Delta$ ex2/3) $[26,27]$.

Large-scale analysis of RNA-seq datasets have been previously performed for TP63 isoforms [28]; however, there was an unmet need to complete a similar analysis for p73 and integrate its expression patterns with p63. Thus, we analyzed p73 expression using human tissue RNA-seq data from the GTEx (Genotype-Tissue Expression) Project $[29,30]$. In parallel, we also analyzed p63 expression to determine if coordinate p73 and p63 expression occurred and compared the protein expression patterns between human and murine tissue. To overcome the challenges of quantifying p73 and p63 mRNA isoforms, we developed an algorithm to analyze exon junction-spanning reads and independently quantified $\mathrm{N}$-terminal and $\mathrm{C}$-terminal isoform expression of p73 and p63. We found that p73 and p63 expression correlated across tissues, with most tissues expressing both genes or neither. We analyzed RNA-seq data to determine the expression of p73 alternative promoter usage isoforms in epithelial tissues and provide evidence suggesting the existence of a novel p73 transcript encoded by exons 4 through 14 . Our analyses are the most extensive to date on $\mathrm{p} 73$ and p63 protein and mRNA expression patterns within normal adult tissues from both humans

\footnotetext{
${ }^{1}$ Department of Biochemistry, Vanderbilt University, Nashville, TN, USA. ${ }^{2}$ Department of Medicine, Vanderbilt University, Nashville, TN, USA. ${ }^{3}$ Vanderbilt-Ingram Cancer Center, Vanderbilt University Medical Center, Nashville, TN, USA. ${ }^{4}$ Department of Pathology, Microbiology and Immunology, Vanderbilt University, Nashville, TN, USA. ${ }^{5}$ Gilead Sciences Inc., Foster, CA, USA. ${ }^{6}$ These authors contributed equally: Clayton B. Marshall, J. Scott Beeler. ${ }^{\bowtie}$ email: j.pietenpol@vumc.org Edited by F. Bernassola
} 
and mice. We observe coordinate expression patterns of p73 and p63 in basal cell populations of epithelial tissues.

\section{RESULTS}

\section{TP73 and TP63 gene expression in human tissue}

To determine the expression patterns of TP73 and TP63 in human tissue, we analyzed RNA-seq data from the GTEx Project $[29,30]$. We observed a significant correlation $\left(r_{\mathrm{s}}=0.49, p=0.0003\right)$ between TP73 and TP63 expression across all tissues and found that TP63 transcript was expressed at higher levels than TP73 in most tissues (Fig. 1A). In our previous work, we observed similar gene expression using RNA-seq data from the Human Protein Atlas [19]. We identified four major groupings of TP73/TP63 expression across tissues. The majority of tissues expressed lowlevels $[<2$ transcripts per million (TPM)] of both TP73 and TP63 (p73-Low/p63-Low) (Fig. 1A, gray shading). The second group of tissues expressed elevated levels (>2 TPM) of both TP73 and TP63 (p73-High/p63-High) (Fig. 1A, yellow shading). A small number of tissues preferentially expressed TP73 (p73-High) (Fig. 1A, purple shading) or TP63 (p63-High) (Fig. 1A, green shading).

\section{Atlas of p73 and p63 protein expression in human and murine tissue}

Previous publications have examined the protein expression patterns of p73 and p63 in select murine and human tissues, including the mammary gland [31], ovary [8, 31, 32], fallopian tube [17], lower respiratory tract $[17,33,34]$, testis $[17,35,36]$, brain $[20,37-41]$, and skin $[19,25,42]$. In a comprehensive approach, we performed dual p73 and p63 immunofluorescence (IF) alongside serial sections stained with hematoxylin and eosin (H\&E) to determine p73 and p63 protein expression patterns in human tissues (Fig. 1A).

Consistent with RNA expression in the p63-High group (Fig. 1A, green shading), we observed p63-positive staining in both skeletal muscle and bladder with little p73 expression in either human (Fig. S1A) and murine (Fig. S2A) tissue. Of note, the expression pattern in skeletal muscle is corroborated by a previous report that observed p63 staining localizes to sarcomere Z-bands [43].

Similarly, the p73-High tissues (Fig. 1A, purple shading) of the cerebellum and fallopian tube displayed p73 expression with minimal or no p63 protein (human: Fig. S1B, mouse: Fig. S2B). Of note, cerebellar Purkinje cells exhibited more cytoplasmic expression of p73, while the granular layer displayed more nuclear staining (Fig. S1B). In the majority of epithelial tissues (esophagus, vagina, cervix, prostate, and salivary gland) from the p73-High/ p63-High group (Fig. 1A, yellow shading), we found that p73 primarily co-localized with p63 in a subset of basally-located epithelial cell nuclei (human: Fig. 1B, mouse: Fig. S2C). The tissues with the highest co-expression levels were squamous epithelial (esophagus, vagina, and cervix). In contrast, the exocrine tissues (salivary, prostate, and mammary) exhibited lower p73 and p63 expression (Fig. 1B). Previous work from our laboratory focused on the role of $p 73$ in the murine dorsal back skin [19]. We expanded on this and performed dual p73 and p63 IF staining on other human (face, gluteus, and abdomen/groin) and murine (dorsal back, toe, ear, and tongue) epithelia, and observed co-expression of p73 in a subset of basally-localized p63-positive cells in all epithelium analyzed (Figs. S1C and S3A).

\section{Alternative C-terminal splicing of TP73 and TP63 in human tissue}

p73 and p63 isoforms have differential transcriptional activity $[1,5,24,25,44]$ and isoform-specific knockout mice display varying phenotypes [20,32, 38, 45-52], consistent with isoformdependent roles for p73 and p63 in tissue development and homeostasis. Quantification of full-length TP73 and TP63 isoform expression using standard methodologies is challenging due to long transcript lengths, low relative expression levels, isoform variation at both $\mathrm{N}$-terminal and $\mathrm{C}$-terminal ends, and the lack of isoform-specific antibodies. To overcome these challenges and determine p73 and p63 isoform expression in human tissue, we developed an algorithm to quantify the mRNA expression of p73 and p63 isoforms across tissue types by analyzing exon junctionspanning reads from RNA-seq data. We quantified p73 and p63 isoform expression for each sample in GTEx using our algorithm, summarizing the results by the mean of each $\mathrm{N}$-terminal and C-terminal isoform per tissue.

We quantified C-terminal isoform expression (resulting from alterative splicing) for TP73 and TP63 by analyzing the number of RNA-seq reads spanning exon-exon junctions from exons 10-14 (Fig. 2A). In all tissues except skeletal muscle, p63a was the predominant (67-97\%) isoform expressed (Fig. 2B and Table S1). p63 $\beta$ was the second most expressed isoform (5-21\%) in epithelial tissues with the highest expression of TP63 (>10 TPM) (Fig. 2B and Table S1). Consistent with a previously reported study [43], skeletal muscle expressed a high percentage $(85 \%)$ of p63y (Fig. 2B and Table S1). p63 $\gamma$ was expressed at much lower relative amounts $(0-24 \%)$ in all other tissues, and p63 8 was expressed at nominal levels in all tissues examined (Fig. 2B and Table S1). Two types of junction-spanning reads were identified between exons 8-9 of TP63 [53]. Across tissues, we found that full-length E8 was the predominant TP63 isoform expressed ( 70\%) and the shortened variant of exon 8 (E8s) was expressed at lower levels ( 30\%) (Fig. 2A and Table S2).

p73 exhibited greater diversity in C-terminal splicing compared to p63 (Fig. 2B and Table S1). p73a was the predominant Cterminal splicing pattern across most tissues (75-94\%) (Fig. 2B and Table S1). Among these same tissues, p73 $\beta$ was the secondhighest expressed (4-31\%) isoform (Fig. 2B and Table S1). We analyzed $\mathrm{p} 73 \gamma$ and $\mathrm{p} 73 \varepsilon$ in tandem (denoted as $\mathrm{y}+\varepsilon$ in Fig. 2B and Table S1) because these isoforms have an exon structure that is difficult to differentiate. Together, the $p 73 \gamma$ and $p 73 \varepsilon$ C-terminal splicing isoforms were the third most expressed isoform in the majority of tissues studied (0-28\%), with the highest percentages of expression found in neuronal tissues from the p73-Low/p63Low group (Fig. 2B and Table S1). Negligible expression (1-3\%) of $\mathrm{p} 73 \delta$ and $\mathrm{p} 73 \zeta$ was detected in a minority of tissues (Fig. 2B and Table S1).

\section{Analysis of p73 and p63 N-terminal mRNA expression in human tissue}

We anticipated that quantification of $\mathrm{N}$-terminal isoform expression (resulting from differential promoter usage [P1-TA; P2- $\triangle N]$ ) of p73 and p63 (Fig. 2A) would be straightforward similar to Cterminal expression. We analyzed TP63 splice junction counts from exon 3 (E3) to exon 4 (E4) and exon $3^{\prime}\left(E 3^{\prime}\right)$ to E4 to determine canonical promoter usage by tissue. $\Delta \mathrm{Np} 63$ was the predominant isoform (80-100\%) in most tissues from the p73-High/p63-High group (Fig. 1A), including the skin, esophageal mucosa, vagina, and prostate (Fig. S4B and Table S4). The testis was an exception and predominately expressed (96\%) TAp63 (Fig. S4B and Table S4). Among the tissues from the p63 ${ }^{\text {High }}$ group (Fig. 1A), the bladder expressed $\triangle N p 63(100 \%)$, and skeletal muscle expressed TAp63 (100\%) (Fig. S4B and Table S4).

While analyzing the expression of individual p73 exons, we observed that the expression level of E4, which is shared by both $\mathrm{TA}$ and $\Delta \mathrm{N}$ p73 isoforms, was disproportionally greater than the combined expression of E3 and E3' in several epithelial tissues from the p73-High/p63-High group (skin, esophageal mucosa, vagina, and prostate) (Fig. $3 \mathrm{~A}$ and Table S3). We did not observe a similar difference in the expression levels of E4 versus E3 plus E3' for TP63 in the same epithelial tissues (Fig. S4A) or either gene in non-epithelial tissues (skeletal muscle and cerebellum) with either high TP73 or TP63 expression (Figs. 3A and S4A, and Table S3). Decreased $5^{\prime}$ RNA-seq coverage can be caused by low-quality or 


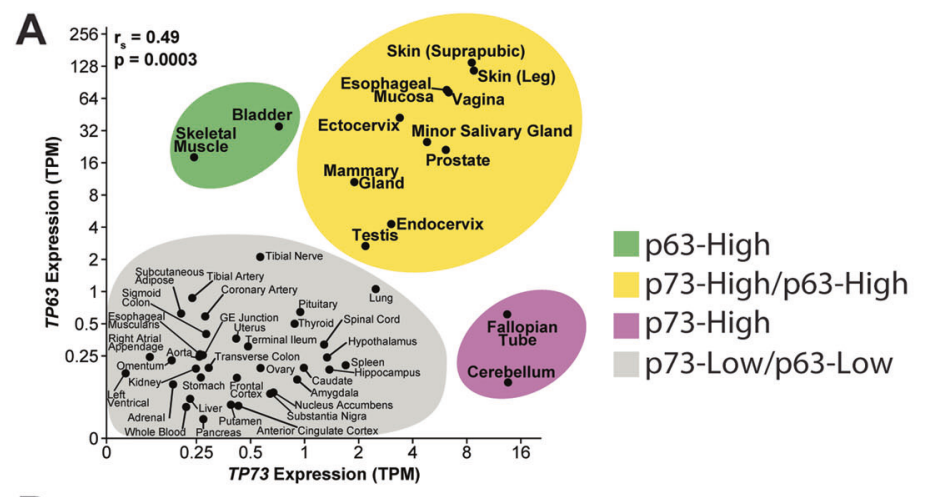

B

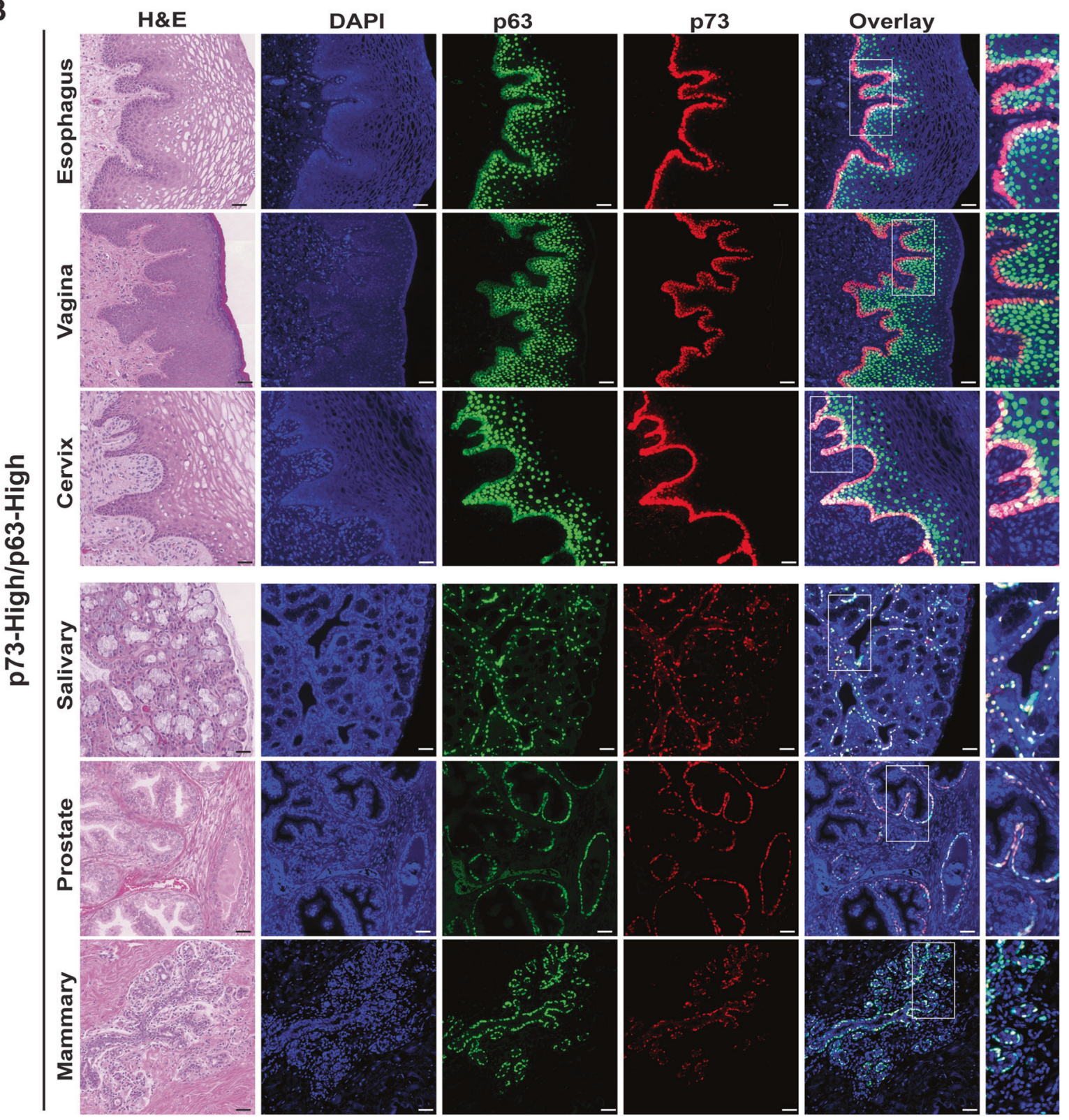

Fig. 1 p73 and p63 Gene and protein expression in human tissue. A TP73 versus TP63 mRNA expression (units = TPM) for each human tissue type in the GTEx dataset. The mean expression (TPM + 0.1) for each tissue is plotted on a $\log _{2}$ scale. Correlation between TP73 and TP63 was quantified using Spearman's rank correlation coefficient $\left(r_{s}\right)$. Tissues were divided into four groups based on the patterns of p73/p63 expression: p73-High/p63-High (yellow shading), p73-High (purple shading), p63-High (green shading), and p73-Low/p63-Low (gray shading). B Representative H\&E and dual IF micrographs (serial sections) from the p73-High/p63-High group described in Part A. p73 (red), p63 (green), and DAPI stained nuclei (blue). For each normal tissue site, samples from three different humans were stained and analyzed to identify the representative area shown. Scale bars $=50 \mu \mathrm{m}$. 


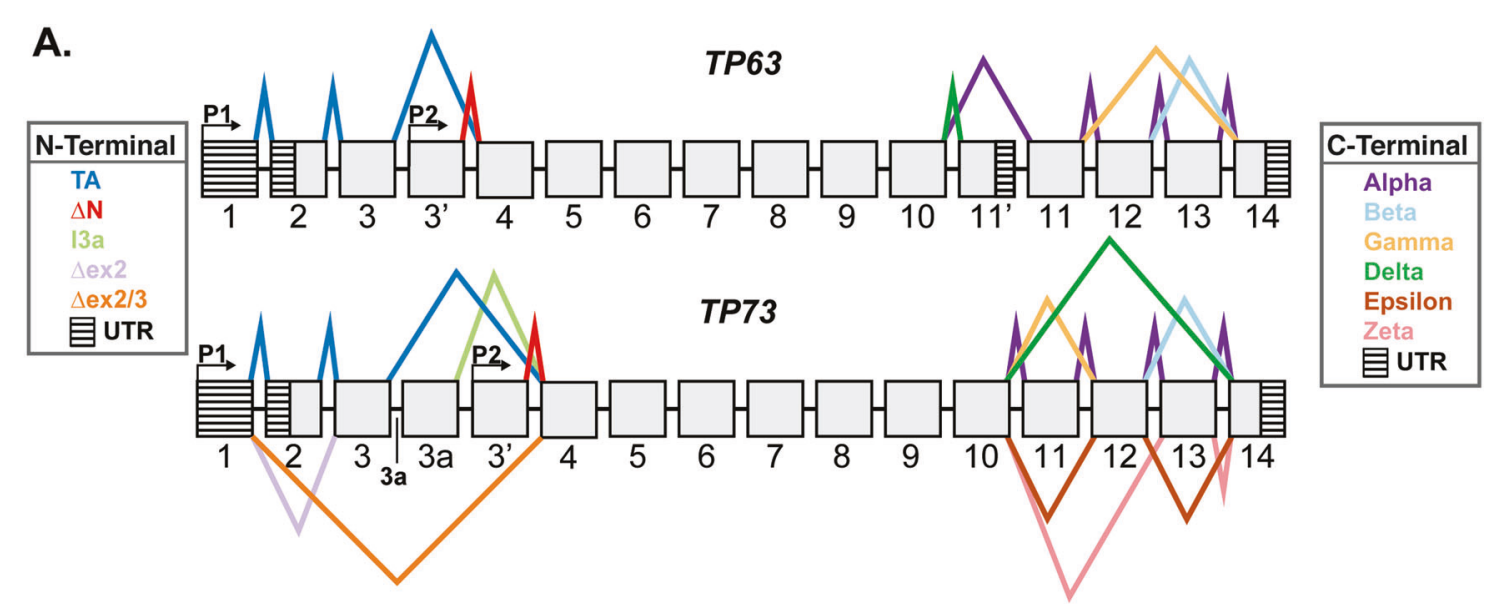

B.



Fig. 2 TP73 and TP63 mRNA isoform expression in human tissue. A Schematic representation of the exon structure and splicing of TP63 and TP73. Promoters (P1-TA and P2- $\Delta \mathrm{N}$ ) are annotated with arrows. Untranslated regions (UTR) are annotated with horizontal stripes. Splicing events are annotated with colored lines, and the key to the line colors is located to the right of the schematic. B Heatmap of RNA-seq expression (TPM) for TP63 and TP73 in human tissue types from the GTEx dataset (left-sided heatmap) with the corresponding relative percentage of C-terminal isoform expression (right-sided heatmaps). Tissues are grouped and shaded according to the key at the bottom of the figure. The number of samples per tissue type can be found in Table S1.

degraded RNA during library preparation, resulting in an inability to generate full-length cDNA during oligo(dT) priming. However, we did not find evidence that samples from epithelial tissues with reduced TP73 E3 + E3' versus E4 expression had lower quality RNA as assessed by RNA integrity number (RIN) or post-mortem interval (time from death to tissue collection) [54, 55]. Also, we analyzed the genomic sequence of TP73 E3'. We determined that it had similar "mappability scores" (a measure of the ability to align 



Fig. 3 TP73 N-terminal Expression in Human Tissue. A Sina plots of TP73 N-terminal exon counts for select human tissue types from the GTEx dataset. Exon counts are normalized by exon length and sequencing depth and plotted on a $\log _{2}$ scale (normalized counts +0.01 ). Each red dot indicates the median value of the population. Tissues are grouped and shaded by the following: p73-High/p63-High (yellow), p73-High (purple), and p63-High (green). The average TP73 gene expression (units = TPM) of each GTEx tissue is listed below its respective plot. B Heatmaps (left-sided) of the mean number of exon junction-spanning reads connecting exon 3 or exon $3^{\prime}$ to exon 4 (E3/3'-E4) and the mean number of junction-spanning reads between exons 5-10 (E5-E10) for both TP73 and TP63. Tissues are grouped and shaded as described in Part A. The right-sided heatmap shows the ratio of E3/E3'-E4 to E5-E10 junction-spanning reads for TP73 and TP63.

short-reads to a sequence) as other exons in the gene (data not shown).

To further evaluate the observed discrepancy in TP73 N-terminal exon expression among epithelial tissues analyzed in Fig. 3A, we turned to isoform junction expression data since it is impacted less by variations in exon length. We calculated the number of exon junction-spanning reads between E3-E4, E3'-E4 and the other exon junctions shared by all canonical p73 isoforms (E5-E10) (Fig. 3B and Table S5). These data were used to calculate the ratio (as a percentage) of the sum of TP73 E3/3'-E4 expression to the mean junction expression of E5-E10 in each tissue (Fig. 3B and Table S5). The same analysis was performed for TP63 for comparison (Fig. 3B and Table S5). Consistent with the exon expression from the p73-High/p63-High group (Fig. 3A), TP73 E3/ $3^{\prime}-$ E4 junctional reads were significantly reduced ( 90\%) compared to the mean of all junctional reads across E5-E10 (Fig. 3B and Table S5). However, in p73-High tissues (cerebellum and fallopian tube), TP73 E3/3'-E4 junctional expression was only reduced by $\sim 30 \%$ compared to E5-E10 (Fig. 3B and Table S5). The calculated ratio percentage for TP73 in the testis is not accurate because the tissue utilizes a unique promoter (I3a) to express an mRNA lacking exons 5-10; thus, we excluded it from the analysis. In line with our prior findings (Fig. 3A), TP63 E3/3'-E4 junction expression was $\sim 75 \%$ of the mean of junctions E5-E10 in tissues from the p73-High/p63-High group (Fig. 3B and Table S5), an amount consistent with the expected degree of $3^{\prime}$ bias typically seen in high-quality RNA-seq experiments utilizing poly-A capture. In sum, these findings suggest that the decrease of TP73 E3/3'-E4 junction expression is specific to epithelial tissue from the p73High/p63-High group, and not merely due to tissue-specific characteristics since a similar reduction in E3/3'-E4 junction expression was not observed for TP63 in these same tissues.

\section{Analysis of TP73 transcription start site usage in human epithelial tissue}

Having identified select epithelial tissues with reduced expression of TP73 E3 and E3', we considered different possibilities for the discordant expression pattern. One such explanation is alternative splicing between exons 1 (E1) or 2 (E2) and E4. GTEx did not report any junctional reads between these exons, and manual review of individual alignments did not find evidence of E1-E4 or E2-E4 junction-spanning reads (data not shown). Another possibility is the existence of an additional transcriptional start site (TSS) in the TP73 locus located at or upstream of E4. Such a transcript would contain an in-frame methionine at the beginning of E4, which could encode for a protein similar in size to $\triangle \mathrm{Np} 73$. To investigate this possibility, we used publicly available $5^{\prime}$-end RNA-seq data for GTEx samples produced by the ENCODE Project [56, 57]. 
These data were generated using the RAMPAGE (RNA Annotation and Mapping of Promoters for the Analysis of Gene Expression) approach, an RNA-seq method to identify TSS at single-base resolution with a high signal-to-noise ratio across the genome [56].

We observed a RAMPAGE peak immediately upstream of TP73 E4 in multiple epithelial tissues from the p73-High/p63-High group, including skin (leg), esophagus, and vagina (Fig. 4A), that would align with an in-frame methionine in E4 (Fig. 4B, arrowhead). Testis also had RAMPAGE read coverage at this peak, but it did not reach genome-wide significance (Fig. 4A). In contrast, the cerebellum, which did not exhibit decreased relative expression of TP73 E3 and E3' (Fig. 3B), lacked read coverage at the peak (Fig. 4A). A RAMPAGE peak was found at the canonical $\triangle N p 73$ TSS ( $5^{\prime}$ end of E3') in only skin (Fig. $4 A$ ). The esophagus and testis also had RAMPAGE reads at this peak that did not achieve genome-wide significance (Fig. 4A). We detected a RAMPAGE peak at the $5^{\prime}$ end of TP73 exon 3a (E3a), specifically in testis (Fig. 4A), the only tissue that expresses significant levels of a transcript composed of only E3a and E4 (data not shown). None of the tissues we investigated had a RAMPAGE peak at the canonical TAp73 TSS at E1 (data not shown). As a control, we analyzed the RAMPAGE peaks of TP63 in the same samples. The only peak detected in TP63 was located at the canonical $\triangle$ Np63 TSS ( $5^{\prime}$ end of E3') and found in skin, esophagus, and vagina (Fig. $\mathrm{S} 5 \mathrm{~A}$ ).

To further assess the potential for a TSS upstream of TP73 E4, we analyzed the RAMPAGE 5 '-end, and RNA-seq reads aligning to this region. In each epithelial tissue with a TP73 E4 peak (Fig. 4A), we found canonical E3-E4 junction-spanning reads in addition to several non-overlapping reads aligning to the intronic region immediately upstream of E4 (Fig. 4B). The maximum length of RNA-seq read coverage upstream of the early methionine in E4 (i.e., putative UTR) was $115 \mathrm{bp}$ for skin, $179 \mathrm{bp}$ for esophagus, and $100 \mathrm{bp}$ for vagina (Fig. 4B). We also analyzed the TP73 read alignments to E3' (Fig. S6A) and confirmed they were consistent with its known role as a UTR and our previous analyses (Figs. $3 \mathrm{~A}$ and $4 \mathrm{~A}$ ). In addition, we analyzed TP73 E7 (Fig. S6B), an exon shared by all isoforms, and ruled out the presence of reads aligning to intronic regions (which can be seen when samples for RNA-seq are contaminated with genomic DNA or pre-mRNA). Lastly, as a control, we performed a corresponding analysis of TP63 E3' and E4 read alignments and did not find any reads aligning to the intronic region upstream of TP63 E4 (Figs. S5 and S7). Collectively, these results provide initial evidence for the existence of a previously unreported (to our knowledge) TP73 mRNA product transcribed in select epithelial tissue from a TSS immediately upstream of E4.

\section{Analysis of Tp73 and Tp63 mRNA in murine tissues}

To extend our p63 and p73 isoform findings to another organism, and analyze primary tissue instead of in silico data sets, we designed appropriate primers as described in "Materials and methods" section and performed qRT-PCR and IF. We analyzed p63 isoform expression in murine skin, vagina, mammary gland, and muscle tissue by qRT-PCR (Fig. S8A). Further, we performed p63 IF on murine vagina and skin tissue (Figs. S2 and S3) and observed congruent protein expression with vagina having the highest level.

Murine TAp73 and $\triangle N p 73$ mRNA expression patterns were similar to those observed in human GTEX data (Fig. S8B). Using maximum shared upstream read length from human tissues as a guide ( 100 bp), we designed murine qRT-PCR primers to evaluate E4p73. We observed expression of a mRNA product corresponding to E4p73 in the murine tissues investigated (Fig. S8B). p73 IF analyses showed that protein expression in murine skin, vagina, mammary gland, and muscle tissue correlates highly with mRNA expression (Figs. S2 and S3).
In summary, p73 and p63 expression are positively correlated across a wide range of normal human and murine tissues. p73 is highly expressed in a subset of p63-positive cells within the epithelial lining of the tissues in which they are co-expressed. As previously reported, p63 is predominately expressed as the $\Delta$ Np63a isoform, while p73 isoform expression is more varied. We have also identified a potential new TSS of p73 that could lead to a protein product that initiates from a methionine in E4.

\section{DISCUSSION}

The analysis of $\mathrm{p} 73$ and $\mathrm{p} 63$ has been challenging due to the lack of isoform-specific antibodies and the large number of isoforms that can be generated from each gene. The development of highthroughput sequencing technologies and their application to transcriptome profiling has made it possible to quantitatively survey the entire transcriptome at single-base resolution [58]. Our analysis of the TP73 and TP63 gene-level expression data from GTEx validated previously published tissue-specific gene expression analyses [5, 16, 28, 59-61]. In addition, the expression patterns of TP73 and TP63 in human tissues, reported herein, aligns with the murine tissues in which phenotypes have been reported using p73-deficient and p63-deficient mice $[17,19,20,31,35,36,38,41,45,48-50,60,62]$.

We were motivated to study the protein expression pattern of p73 and p63 because of the physical and functional interactions between the two transcription factors, and the lack of a comprehensive atlas of their co-expression and localization across tissues. Given the known roles of both p73 and p63 in stem cell regulation, the high co-expression of both proteins in the basal layer of epithelial tissues (e.g., skin, esophagus, vagina, endocervix, and ectocervix) is of interest. Despite the lack of an overt skin phenotype in p73 knockout mice, we recently found that p73 is important in the process of timely epidermal wound healing [19]. Thus, we hypothesize that additional phenotypes would be observed in barrier epithelial tissues with high levels of p73 expression after damage.

Our study has provided initial evidence supporting the existence of a previously unreported p73 mRNA product in multiple epithelial tissues beginning in the intron upstream of E4. A previous study confirmed that the $\mathrm{E} 4$ start codon in $\Delta \mathrm{Np} 73 \mathrm{a}$ could initiate translation and produce a $\Delta N p 73$-like protein product using in vitro translation and cell-based overexpression studies [63]. Further, studies in small cell lung cancer and ovarian carcinoma have observed increased translation initiation from the methionine in E4 in connection to alternative splicing of TAp73, $\Delta$ ex2p73, and $\Delta$ ex2/3p73 $[64,65]$. We did not observe splicing of E4 to either E2 or E1 in the GTEx data; rather, we observed a unique TSS in the intronic region directly upstream of E4, potentially generating a putative UTR upstream of an early E4 methionine. E4 initiation was previously reported for p63 when a truncated $\Delta N p 63$ protein was produced by translation re-initiation at the first methionine in E4 in three patients with an ankyloblepharon-ectodermal dysplasia-cleft lip/palate-like syndrome [66]. It will be important to determine the relative expression level and biological differences between $\Delta \mathrm{Np} 73$ and E4p73 in physiologically-relevant contexts. The first 13 amino acids of $\Delta \mathrm{Np} 73$ isoforms are important in transcriptional activation of downstream target genes [9]. Since E4p73 lacks the first 22 amino acids shared in $\triangle N p 73$, we predict that E4p73 would have reduced transcriptional activity compared to $\Delta \mathrm{Np} 73$ due to the absence of the novel activation domain [9]. Further work is also needed to determine how E4p73 alters TP73 function since most tissues express multiple $\mathrm{N}$-terminal isoforms.

To our knowledge, this study provides the most extensive bioinformatic analysis of TP73 and TP63 mRNA isoform expression across human tissues to date coupled with an atlas of protein expression patterns at the cellular level in humans and mice. 
A

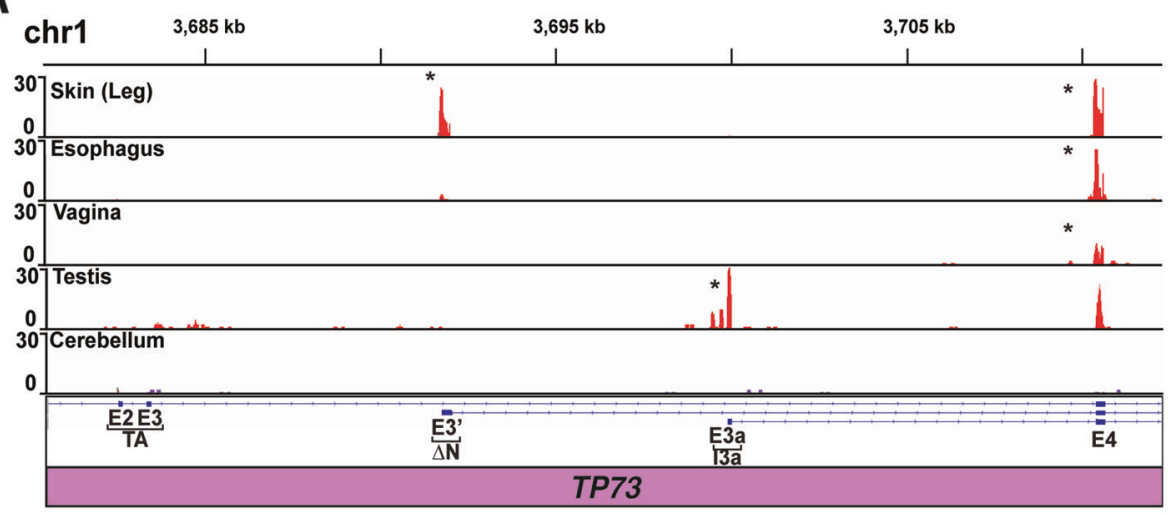

B



Fig. 4 Differential TP73 TSS usage in human tissue. A Genome browser view of the $5^{\prime}$ end of the TP73 genomic locus showing the read density of $5^{\prime}$-end RNA-seq (RAMPAGE) for select human tissue from the ENCODE Project. RAMPAGE peaks (i.e., TSS) were identified using the ENCODE standard RAMPAGE pipeline and are marked with an asterisk. B Genome browser view of TP73 exon 4 showing aligned reads from $5^{\prime}-$ end (RAMPAGE, red-colored reads) and poly-A (GTEx, blue-colored reads) RNA-seq performed on the same skin, esophagus, and vagina samples. The thin gray lines annotate reads aligning to the junctions between exon 3 or $3^{\prime}$ and exon 4 . The methionine within exon 4 (green colored, marked with an arrowhead) is the putative start codon of E4p73. 
Given the rigorous procedures for tissue procurement and RNAseq used by the GTEx Project [67], the analyses herein should be useful to investigators interested in tissue-specific expression and the functional relevance of the various p73 and p63 isoforms. The results presented herein provide further evidence that the study of p73 and p63 in isolation offers only a partial view of potential signaling outputs, as the proteins are co-expressed in a wide array of cell types and can heterodimerize. Future studies should investigate both proteins in concert to understand their independent and dependent functions better.

\section{MATERIALS AND METHODS \\ Human and murine sample acquisition}

Human tissue sections were selected by a board-certified pathologist (M.E. S) under the auspices of IRB \# 192076, "Normal Tissue Atlas." Murine studies described herein were carried out according to recommendations in the Guide for the Care, and Use of Laboratory Animals of the National Institutes of Health (NIH), and protocols used were approved by the Institutional Animal Care and Use Committee (IACUC) of Vanderbilt University Medical Center (VUMC) (Protocol Number: M1800069). All IF staining of human tissue was conducted in triplicate on samples from at least three different individuals. Murine IF experiments were conducted in male and female mice from BALB/C and C57BL/6 backgrounds in triplicate to ensure staining results were not biased by gender or strain.

\section{Immunofluorescence (IF)}

Immunostaining of tissue sections was performed as previously described [17]. IF was conducted using the antibodies p73 EP436Y (Abcam ab40658; 1:1000; Cambridge, UK) and p63a D2K8X (Cell Signaling Technology \#13109; 1:1000; Danvers, MA), with TSA Plus Fluorescence Amplification Kit (Perkin Elmer, Waltham MA). Nuclei were counterstained with Slowfade Gold DAPI mounting media (Invitrogen, Carlsbad, CA). Micrographs of human tissues were all taken using a Zeiss slide scanner and further analyzed using Zeiss software. Micrographs of murine tissues were taken using a Leica microscope and camera and analyzed using Leica software; all scale bars $=50 \mu \mathrm{m}$.

\section{Genotype-tissue expression (GTEx) RNA-seq analysis}

RNA-seq data for 11,688 human samples (51 normal tissue sites and two primary cell lines) was downloaded from the GTEx Portal on January 1, 2018 (V7 Release) [67, 68]. The RNA-seq libraries were generated from total RNA using the Illumina TruSeq RNA Library Preparation Kit (non-stranded, poly-A capture) and sequenced on the Illumina HiSeq 2000 or $2500(>50$ million 76 base pair paired-end reads per sample). Sequencing reads were aligned to hg19 with STAR v2.4.2a [69] using GENCODE v19 [70] annotations. Gene-level expression (units = TPM) were quantified using RNA-SeQC v1.1.8 [71] and junction read counts using STAR v2.4.2a [69]. For each sample, the number of reads spanning each TP73 and TP63 exon-exon junction was calculated, normalized to the counts per million (read counts/total number of aligned reads/1e6), and used to determine isoform expression for the $\mathrm{N}$-terminus and $\mathrm{C}$-terminus independently. The relative amount of normalized junction counts for exon 3 to exon 4 (E3-E4; corresponds to the TA isoform) versus exon $3^{\prime}$ to exon 4 (E3-E4; corresponds to the $\Delta \mathrm{N}$ isoform) was used to determine the percentage of $\mathrm{N}$-terminal isoform expression for both genes. For TP73, the relative amount of exon 3a to exon 4 (E3a-E4; corresponds to the I3a isoform) was also incorporated into the analysis. The GTEx junction count quantification data set did not contain information on the exon-spanning junctions corresponding to three previously identified N-terminal isoforms (Ex2p73, Ex2/3p73, and $\Delta N p 73^{\prime}$ ) [72]. Junction counts for TP63 corresponding to exon 4 skipping (exon 3 or exon $3^{\prime}$ to exon 5) and an additional 216 base pair exon (located in the intron between exons 4 and 5) were detected at very low levels and thus excluded from the analysis (lacked tissue-specific expression, their expression simply correlated with total TP63 expression). C-terminal isoform expression for both genes was calculated similarly with slight modifications for each gene to account for differences in alternative splicing between them. The percentage of $p 73 a+\beta$ [exon 10 to exon 11 (E10-E11)], p73 $\gamma+\varepsilon$ [exon 10 to exon 12 (E10-E12)], p73Z [exon 10 to exon 13 (E10-E13)], and p738 [exon 10 to exon 14 (E10-E14)] was calculated by analyzing normalized exon-exon junction counts starting at exon 10 (E10). The relative expression of $p 73 a$ versus $\beta$ was determined by comparing normalized exon 12 to exon 13 (E12-E13; corresponds to the a isoform) versus exon 12 to exon 14 (E12-E14; corresponds to the $\beta$ isoform) junction counts. The percentage of p63y [exon 10 to exon 11a (E10-E11a)] versus non-p63y (E10-E11) was calculated by analyzing normalized exon-exon junction counts starting at E10. The percentage of p638 [exon 11 to exon 14 (E11-E14)] versus non-p638 (E11-E12) expression was calculated, including normalization for the previously determined percentage of p63 $\gamma$ expression (multiplied relative percentages of $p 63 \delta$ and nonp63 $\delta$ by the non-p63y from the prior calculation). The percentage of $p 63 \beta$ (E12-E14) and p63a (E12-E13) was calculated using the same methodology as p638. Isoform expression for each tissue was calculated as the mean isoform expression for all samples belonging to a given tissue. We calculated the ratio (as a percentage) of the E3/3'-E4 junction expression to mean junction expression of E5-E10 for TP73 and TP63 in each tissue using the following equation: (sum of E3/3'-E4 normalized expression)/(mean normalized expression of exon junctions between E5-E10) $\times 100$. A total of 36 tissues were included in the final results (Table S1). The GTEx ID's of individual samples we show in Fig. 4 were Skin (Leg) (GTEX-1GF9X-1326SM-7PC2Y), Esophagus (GTEX-1LGRB-1626-SM-CNNR7) and Vagina (GTEX1HCUA-2426-SM-ADEIS).

\section{Encyclopedia of DNA elements (ENCODE) TSS 5'-end RNA-seq analysis}

RAMPAGE (RNA Annotation and Mapping of Promoters for the Analysis of Gene Expression) $[56,57]$ data for human adult and embryonic tissues with TP73, and/or TP63 expression $(n=17)$ was downloaded from the ENCODE Project Portal [73] on December 8, 2019. TSS identified by the ENCODE RAMPAGE pipeline were manually reviewed in IGV $[74,75]$ for each sample to validate that the signal for unique reads at the TP73 and TP63 genomic loci was consistent with TSS calls. The ENCODE ID's of individual samples shown in Fig. 4 are: Skin (Leg) (ENCFF174CGG), Esophagus (ENCFF970OJS), Vagina (ENCFF838AHK), Prostate (ENCFF362JKL), and Cerebellum (ENCFF569IAA).

\section{Murine RNA preparation and qRT-PCR analysis}

RNA was harvested from tissue by homogenizing murine muscle, mammary, vagina, and dorsal skin samples in Trizol and purified using the Aurum Total RNA Mini kit (Bio-Rad) in triplicate. qRT-PCR experiments were conducted using oligo(dT)-mediated first-strand synthesis and SYBR Green quantification. mRNA levels were quantified using primers targeting TAp73 (exon 3-4 junction) (TAp73 Forward: 5' GTTGGGGAGATGGCCCAGA CCT 3'/TAp73 Reverse: 5' CCATGTTGGACTCCTCGCTGCC 3'), $\Delta$ Np73 (exon $3^{\prime}-4$ junction) ( $\triangle$ Np73 Forward: 5' CACACCAGCTCCTCAGCGTGTG $3^{\prime} / \Delta$ Np73 Reverse: $5^{\prime}$ CTGGTCCATGGCACTGCTGAGC 3') E4p73 (Exon4) (E4p73 Forward: 5' TGTTTCTCCCCTCCCACCTCCC 3' /E4p73 Reverse: 5' CCCATCTGG TCCATGGCACTGC $3^{\prime}$ ) and TAp63 (exon 3-4 junction) (TAp63 Forward: $5^{\prime}$ TGCCACCCTACAGTACTGCCCC 3'/TAp63 Reverse: 5' CTCGCTTGTCTGGGTG CTCTGC $3^{\prime}$ ), $\Delta$ Np63 (exon $3^{\prime}-4$ junction) ( $\triangle$ Np63 Forward: $5^{\prime}$ GCAGCCTT GACCAGTCTCACTGC $3^{\prime} / \Delta$ Np63 Reverse: 5' TCCATGCTGTTCAGGAGCCCCA $\left.3^{\prime}\right)$. All of the isoform specific qRT-PCR levels were normalized to the expression level of GAPDH using the primers (GGGGTCGTTGATGGCAACA/ AGGTCGGTGTGAACGGATTTG).

\section{Statistical analysis}

All statistical analyses and graphical representations were conducted using $\mathrm{R}$ (version 3.5 .2 or 3.5.3) unless otherwise noted. The Spearman correlation between TP63 and TP73 expression across human tissues (GTEx RNA-seq dataset), and its statistical significance were calculated using the "cor.test" function in $R$.

\section{CODE AVAILABILITY}

All code used for the analysis of GTEx data can be found on Figshare (https://doi.org/ 10.6084/m9.figshare.14684190). File "20210526-GTEx_data_processing.sh" contains the code used to process GTEx RNA-seq data in order to obtain mRNA expression data for TP73 and TP63. The file "20210526-key_computational_analyses.R" contains the code used to quantify and graph TP73 and TP63 mRNA expression at the gene, exon, and isoform level. 


\section{REFERENCES}

1. Kaghad M, Bonnet H, Yang A, Creancier L, Biscan JC, Valent A, et al. Monoallelically expressed gene related to $p 53$ at $1 p 36$, a region frequently deleted in neuroblastoma and other human cancers. Cell 1997;90:809-19.

2. Osada M, Ohba M, Kawahara C, Ishioka C, Kanamaru R, Katoh I, et al. Cloning and functional analysis of human $\mathrm{p} 51$, which structurally and functionally resembles p53. Nat Med. 1998;4:839-43.

3. Schmale $\mathrm{H}$, Bamberger $\mathrm{C}$. A novel protein with strong homology to the tumor suppressor p53. Oncogene1997;15:1363-7.

4. Trink B, Okami K, Wu L, Sriuranpong V, Jen J, Sidransky D. A new human p53 homologue. Nat Med. 1998;4:747-8.

5. Yang A, Kaghad M, Wang $Y$, Gillett $E$, Fleming MD, Dotsch $V$, et al. p63, a p53 homolog at 3q27-29, encodes multiple products with transactivating, deathinducing, and dominant-negative activities. Mol Cell1998;2:305-16.

6. el-Deiry WS, Kern SE, Pietenpol JA, Kinzler KW, Vogelstein B. Definition of a consensus binding site for p53. Nat Genet. 1992;1:45-9.

7. Cai BH, Chao CF, Huang HC, Lee HY, Kannagi R, Chen JY. Roles of p53 family structure and function in non-canonical response element binding and activation. Int J Mol Sci. 2019;20:15.

8. Crum CP, McKeon FD. p63 in epithelial survival, germ cell surveillance, and neoplasia. Annu Rev Pathol. 2010;5:349-71.

9. Liu G, Nozell S, Xiao H, Chen X. DeltaNp73beta is active in transactivation and growth suppression. Mol Cell Biol. 2004;24:487-501.

10. Osada M, Park HL, Nagakawa Y, Yamashita K, Fomenkov A, Kim MS, et al. Differential recognition of response elements determines target gene specificity for p53 and p63. Mol Cell Biol. 2005;25:6077-89.

11. Zhu J, Jiang J, Zhou W, Chen X. The potential tumor suppressor p73 differentially regulates cellular p53 target genes. Cancer Res. 1998;58:5061-5.

12. Davison TS, Vagner C, Kaghad M, Ayed A, Caput D, Arrowsmith CH. p73 and p63 are homotetramers capable of weak heterotypic interactions with each other but not with p53. J Biol Chem. 1999;274:18709-14.

13. Rocco JW, Leong CO, Kuperwasser N, DeYoung MP, Ellisen LW. p63 mediates survival in squamous cell carcinoma by suppression of p73-dependent apoptosis. Cancer Cell 2006;9:45-56.

14. Friedman PN, Chen X, Bargonetti J, Prives C. The p53 protein is an unusually shaped tetramer that binds directly to DNA. Proc Natl Acad Sci USA. 1993;90:3319-23.

15. Clore GM, Omichinski JG, Sakaguchi K, Zambrano N, Sakamoto H, Appella E, et al. High-resolution structure of the oligomerization domain of $\mathrm{p} 53$ by multidimensional NMR. Science 1994;265:386-91.

16. Puig P, Capodieci P, Drobnjak M, Verbel D, Prives C, Cordon-Cardo C, et al. p73 Expression in human normal and tumor tissues: loss of p73alpha expression is associated with tumor progression in bladder cancer. Clin Cancer Res. 2003;9:5642-51.

17. Marshall CB, Mays D, Beeler JS, Rosenbluth JM, Boyd KL, Santos Guasch GL, et al. p73 is required for multiciliogenesis and regulates the Foxj1-associated gene network. Cell Rep. 2016;14:2289-300.

18. Sada A, Jacob F, Leung E, Wang S, White BS, Shalloway D, et al. Defining the cellular lineage hierarchy in the interfollicular epidermis of adult skin. Nat Cell Biol. 2016;18:619-31.

19. Beeler JS, Marshall CB, Gonzalez-Ericsson PI, Shaver TM, Santos Guasch GL, Lea ST, et al. p73 regulates epidermal wound healing and induced keratinocyte programming. PLoS ONE. 2019;14:e0218458.

20. Yang A, Walker N, Bronson R, Kaghad M, Oosterwegel M, Bonnin J, et al. p73deficient mice have neurological, pheromonal and inflammatory defects but lack spontaneous tumours. Nature 2000;404:99-103.

21. Dohn M, Zhang S, Chen X. p63alpha and DeltaNp63alpha can induce cell cycle arrest and apoptosis and differentially regulate p53 target genes. Oncogene 2001;20:3193-205.

22. Wu G, Nomoto S, Hoque MO, Dracheva T, Osada M, Lee CC, et al. DeltaNp63alpha and TAp63alpha regulate transcription of genes with distinct biological functions in cancer and development. Cancer Res. 2003;63:2351-7.

23. Pokorna Z, Vyslouzil J, Hrabal V, Vojtesek B, Coates PJ. The foggy world(s) of p63 isoform regulation in normal cells and cancer. J Pathol. 2021;254:454-73.

24. De Laurenzi V, Costanzo A, Barcaroli D, Terrinoni A, Falco M, AnnicchiaricoPetruzzelli $M$, et al. Two new p73 splice variants, gamma and delta, with different transcriptional activity. J Exp Med. 1998;188:1763-8.

25. De Laurenzi V, Rossi A, Terrinoni A, Barcaroli D, Levrero M, Costanzo A, et al. p63 and p73 transactivate differentiation gene promoters in human keratinocytes. Biochem Biophys Res. Commun. 2000;273:342-6.

26. Fillippovich I, Sorokina N, Gatei M, Haupt $\mathrm{Y}$, Hobson $\mathrm{K}$, Moallem $\mathrm{E}$, et al Transactivation-deficient p73alpha (p73Deltaexon2) inhibits apoptosis and competes with p53. Oncogene 2001;20:514-22.

27. Ishimoto O, Kawahara C, Enjo K, Obinata M, Nukiwa T, Ikawa S. Possible oncogenic potential of DeltaNp73: a newly identified isoform of human p73. Cancer Res. 2002;62:636-41.
28. Sethi I, Romano RA, Gluck C, Smalley K, Vojtesek B, Buck MJ, et al. A global analysis of the complex landscape of isoforms and regulatory networks of p63 in human cells and tissues. BMC Genomics. 2015;16:584.

29. Consortium GT, Laboratory DA. Coordinating Center-Analysis Working G, Statistical Methods groups-Analysis Working G, Enhancing Gg, Fund NIHC, et al. Genetic effects on gene expression across human tissues. Nature. 2017; 550:204-13.

30. Carithers LJ, Moore HM. The genotype-tissue expression (GTEx) project. Biopreserv Biobank. 2015;13:307-8.

31. Santos Guasch GL, Beeler JS, Marshall CB, Shaver TM, Sheng Q, Johnson KN, et al. p73 is required for ovarian follicle development and regulates a gene network involved in cell-to-cell adhesion. iScience. 2018;8:236-49.

32. Suh EK, Yang A, Kettenbach A, Bamberger C, Michaelis AH, Zhu Z, et al. p63 protects the female germ line during meiotic arrest. Nature 2006;444:624-8.

33. Nemajerova A, Kramer D, Siller SS, Herr C, Shomroni O, Pena T, et al. TAp73 is a central transcriptional regulator of airway multiciliogenesis. Genes Dev. 2016:30:1300-12.

34. Daniely Y, Liao G, Dixon D, Linnoila Rl, Lori A, Randell SH, et al. Critical role of p63 in the development of a normal esophageal and tracheobronchial epithelium. Am J Physiol Cell Physiol. 2004;287:C171-81.

35. Holembowski L, Kramer D, Riedel D, Sordella R, Nemajerova A, Dobbelstein M, et al. TAp73 is essential for germ cell adhesion and maturation in testis. J Cell Biol. 2014;204:1173-90.

36. Inoue S, Tomasini R, Rufini A, Elia AJ, Agostini M, Amelio I, et al. TAp73 is required for spermatogenesis and the maintenance of male fertility. Proc Natl Acad Sci USA. 2014;111:1843-8.

37. Fuertes-Alvarez S, Maeso-Alonso L, Villoch-Fernandez J, Wildung M, Martin-Lopez $M$, Marshall $C$, et al. p73 regulates ependymal planar cell polarity by modulating actin and microtubule cytoskeleton. Cell Death Dis. 2018;9:1183.

38. Wilhelm MT, Rufini A, Wetzel MK, Tsuchihara K, Inoue S, Tomasini R, et al. Isoformspecific p73 knockout mice reveal a novel role for delta Np73 in the DNA damage response pathway. Genes Dev. 2010;24:549-60.

39. Agostini $M$, Tucci $P$, Chen $H$, Knight RA, Bano $D$, Nicotera $P$, et al. p73 regulates maintenance of neural stem cell. Biochem Biophys Res. Commun. 2010;403:13-7.

40. Alexandrova EM, Talos F, Moll UM. p73 is dispensable for commitment to neura stem cell fate, but is essential for neural stem cell maintenance and for blocking premature differentiation. Cell Death Differ. 2013;20:368.

41. Talos F, Abraham A, Vaseva AV, Holembowski L, Tsirka SE, Scheel A, et al. p73 is an essential regulator of neural stem cell maintenance in embryonal and adult CNS neurogenesis. Cell Death Differ. 2010;17:1816-29.

42. Botchkarev VA, Flores ER. p53/p63/p73 in the epidermis in health and disease Cold Spring Harb Perspect Med. 2014;4:a015248.

43. Martin SE, Temm CJ, Goheen MP, Ulbright TM, Hattab EM. Cytoplasmic p63 immunohistochemistry is a useful marker for muscle differentiation: an immunohistochemical and immunoelectron microscopic study. Mod Pathol. 2011;24:1320-6.

44. De Laurenzi VD, Catani MV, Terrinoni A, Corazzari M, Melino G, Costanzo A, et al. Additional complexity in p73: induction by mitogens in lymphoid cells and identification of two new splicing variants epsilon and zeta. Cell Death Differ. 1999;6:389-90.

45. Niklison-Chirou MV, Steinert JR, Agostini M, Knight RA, Dinsdale D, Cattaneo A, et al. TAp73 knockout mice show morphological and functional nervous system defects associated with loss of p75 neurotrophin receptor. Proc Natl Acad Sci USA. 2013;110:18952-7.

46. Rufini A, Niklison-Chirou MV, Inoue S, Tomasini R, Harris IS, Marino A, et al. TAp73 depletion accelerates aging through metabolic dysregulation. Genes Dev. 2012;26:2009-14.

47. Tomasini R, Tsuchihara K, Tsuda C, Lau SK, Wilhelm M, Ruffini A, et al. TAp73 regulates the spindle assembly checkpoint by modulating BubR1 activity. Proc Natl Acad Sci USA. 2009;106:797-802.

48. Tomasini R, Tsuchihara K, Wilhelm M, Fujitani M, Rufini A, Cheung CC, et al. TAp73 knockout shows genomic instability with infertility and tumor suppressor functions. Genes Dev. 2008;22:2677-91.

49. Mills AA, Zheng B, Wang XJ, Vogel H, Roop DR, Bradley A. p63 is a p53 homologue required for limb and epidermal morphogenesis. Nature 1999;398:708-13.

50. Yang A, Schweitzer R, Sun D, Kaghad M, Walker N, Bronson RT, et al. p63 is essential for regenerative proliferation in limb, craniofacial and epithelial development. Nature 1999:398:714-8.

51. Guo X, Keyes WM, Papazoglu C, Zuber J, Li W, Lowe SW, et al. TAp63 induces senescence and suppresses tumorigenesis in vivo. Nat Cell Biol. 2009;11:1451-7.

52. Su X, Paris M, Gi YJ, Tsai KY, Cho MS, Lin YL, et al. TAp63 prevents premature aging by promoting adult stem cell maintenance. Cell Stem Cell. 2009;5:64-75.

53. Rizzo JM, Romano RA, Bard J, Sinha S. RNA-seq studies reveal new insights into p63 and the transcriptomic landscape of the mouse skin. J Invest Dermatol. 2015;135:629-32. 
54. Ferreira PG, Munoz-Aguirre M, Reverter F, Sa Godinho CP, Sousa A, Amadoz A, et al. The effects of death and post-mortem cold ischemia on human tissue transcriptomes. Nat Commun. 2018;9:490.

55. Consortium G. GTExPortal. https://www.gtexportal.org/home/documentationPage.

56. Batut $P$, Gingeras TR. RAMPAGE: promoter activity profiling by paired-end sequencing of 5'-complete cDNAs. Curr Protoc Mol Biol. 2013;104:25B-11.

57. Batut $P$, Dobin A, Plessy C, Carninci P, Gingeras TR. High-fidelity promoter profiling reveals widespread alternative promoter usage and transposon-driven developmental gene expression. Genome Res. 2013;23:169-80.

58. Wang Z, Gerstein M, Snyder M. RNA-Seq: a revolutionary tool for transcriptomics. Nat Rev Genet. 2009;10:57-63.

59. Grespi F, Amelio I, Tucci P, Annicchiarico-Petruzzelli M, Melino G. Tissue-specific expression of p73 C-terminal isoforms in mice. Cell Cycle 2012;11:4474-83.

60. Conforti F, Yang AL, Agostini M, Rufini A, Tucci P, Nicklison-Chirou MV, et al. Relative expression of TAp73 and DeltaNp73 isoforms. Aging. 2012;4:202-5.

61. Di Como CJ, Urist MJ, Babayan I, Drobnjak M, Hedvat CV, Teruya-Feldstein J, et al. p63 expression profiles in human normal and tumor tissues. Clin Cancer Res. 2002;8:494-501.

62. Holembowski L, Schulz R, Talos F, Scheel A, Wolff S, Dobbelstein M, et al. While p73 is essential, p63 is completely dispensable for the development of the central nervous system. Cell Cycle. 2011;10:680-9.

63. Grob TJ, Novak U, Maisse C, Barcaroli D, Luthi AU, Pirnia F, et al. Human delta Np73 regulates a dominant negative feedback loop for TAp73 and p53. Cell Death Differ. 2001;8:1213-23.

64. George J, Lim JS, Jang SJ, Cun Y, Ozretic L, Kong G, et al. Comprehensive genomic profiles of small cell lung cancer. Nature 2015;524:47-53.

65. Concin N, Becker K, Slade N, Erster S, Mueller-Holzner E, Ulmer H, et al. Transdominant DeltaTAp73 isoforms are frequently up-regulated in ovarian cancer. Evidence for their role as epigenetic p53 inhibitors in vivo. Cancer Res. 2004;64:2449-60.

66. Rinne T, Clements SE, Lamme E, Duijf PH, Bolat E, Meijer R, et al. A novel translation re-initiation mechanism for the p63 gene revealed by amino-terminal truncating mutations in Rapp-Hodgkin/Hay-Wells-like syndromes. Hum Mol Genet. 2008;17:1968-77.

67. Carithers LJ, Ardlie K, Barcus M, Branton PA, Britton A, Buia SA, et al. A novel approach to high-quality postmortem tissue procurement: the GTEx project. Biopreserv Biobank. 2015;13:311-9.

68. Consortium GT. The genotype-tissue expression (GTEx) project. Nat Genet. 2013;45:580-5.

69. Dobin A, Davis CA, Schlesinger F, Drenkow J, Zaleski C, Jha S, et al. STAR: ultrafast universal RNA-seq aligner. Bioinformatics 2013;29:15-21.

70. Harrow J, Frankish A, Gonzalez JM, Tapanari E, Diekhans M, Kokocinski F, et al. GENCODE: the reference human genome annotation for The ENCODE Project. Genome Res. 2012;22:1760-74.

71. DeLuca DS, Levin JZ, Sivachenko A, Fennell T, Nazaire MD, Williams C, et al. RNASeQC: RNA-seq metrics for quality control and process optimization. Bioinformatics 2012;28:1530-2

72. Murray-Zmijewski F, Lane DP, Bourdon JC. p53/p63/p73 isoforms: an orchestra of isoforms to harmonise cell differentiation and response to stress. Cell Death Differ. 2006;13:962-72.

73. Davis CA, Hitz BC, Sloan CA, Chan ET, Davidson JM, Gabdank I, et al. The Encyclopedia of DNA elements (ENCODE): data portal update. Nucleic Acids Res. 2018:46:D794-D801.

74. Thorvaldsdottir H, Robinson JT, Mesirov JP. Integrative Genomics Viewer (IGV): high-performance genomics data visualization and exploration. Brief Bioinform. 2013;14:178-92.

75. Robinson JT, Thorvaldsdottir H, Winckler W, Guttman M, Lander ES, Getz G, et al. Integrative genomics viewer. Nat Biotechnol. 2011;29:24-6.

\section{ACKNOWLEDGEMENTS}

We thank members of the Pietenpol laboratory for their critical review of this manuscript. This research was supported by National Institutes of Health (NIH) grants
R01CA105436, R01CA070856, P50CA098131, and P30CA068485 to JAP and NIH Vanderbilt Medical Scientist Training Program (T32GM007347) training grant support provided to JSB.

\section{AUTHOR CONTRIBUTIONS}

CBM conceived and designed the analysis, conducted all immunofluorescencerelated experiments, and wrote the manuscript. JSB conceived and designed the analysis, conducted bioinformatics-related analyses of the GTEx, and RAMPAGE data, and helped write the manuscript. BDL assisted with creating heatmaps from bioinformatic analyses, as well as manuscript and figure editing. PGE assisted with the analysis of human tissue staining, as well as manuscript and figure editing. VS assisted with the acquisition of human tissue samples and their sectioning, as well as manuscript and figure editing. MES assisted with the acquisition of human tissue samples, as well as manuscript and figure editing. KLB assisted with murine sample analysis, as well as manuscript and figure editing. JAP provided significant contributions to the design, analysis, and interpretation of data, as well as editing and final approval of the finalized manuscript for publication.

\section{ETHICS STATEMENT}

Human tissue sections were selected by a board-certified pathologist, Melinda E. Sanders under the auspices of IRB \# 192076, "Normal Tissue Atlas". All murine studies described herein were carried out according to recommendations in the Guide for the Care, and Use of Laboratory Animals of the National Institutes of Health (NIH), and protocols used were approved by the Institutional Animal Care and Use Committee (IACUC) of Vanderbilt University Medical Center (VUMC) (Protocol Number: M1800069).

\section{COMPETING INTERESTS}

The authors declare no competing interests.

\section{ADDITIONAL INFORMATION}

Supplementary information The online version contains supplementary material available at https://doi.org/10.1038/s41419-021-04017-8

Correspondence and requests for materials should be addressed to J.A.P.

Reprints and permission information is available at http://www.nature.com/ reprints

Publisher's note Springer Nature remains neutral with regard to jurisdictional claims in published maps and institutional affiliations.



Open Access This article is licensed under a Creative Commons Attribution 4.0 International License, which permits use, sharing, adaptation, distribution and reproduction in any medium or format, as long as you give appropriate credit to the original author(s) and the source, provide a link to the Creative Commons license, and indicate if changes were made. The images or other third party material in this article are included in the article's Creative Commons license, unless indicated otherwise in a credit line to the material. If material is not included in the article's Creative Commons license and your intended use is not permitted by statutory regulation or exceeds the permitted use, you will need to obtain permission directly from the copyright holder. To view a copy of this license, visit http://creativecommons. org/licenses/by/4.0/.

(c) The Author(s) 2021 